\title{
ANALYSIS OF LIPOPHILIC FRACTION FROM LEAVES, INFLORESCENCES AND RHIZOMES OF SILPHIUM PERFOLIATUM L.
}

\author{
RADOSŁAW KOWALSKI \\ Central Apparatus Laboratory, Agricultural University \\ Sub-department of Instrumental Foodstuff Analysis, \\ Agricultural University \\ Akademicka 13, 20-950 Lublin, Poland \\ e-mail: radko@ursus.ar.lublin.pl
}

(Received: June 11, 2004. Accepted: November 2, 2004)

\begin{abstract}
This paper presents a qualitative and quantitative chromatographic analysis (GC/MS) of the lipophilic fraction extracted from leaves, inflorescences and rhizomes of Silphium perfoliatum L. collected in 1998-2000.

It was shown that the extracts of leaves and inflorescences are similar in composition, containing among their main constituents: $\alpha$-amyrine (up to $13.9 \%$ of total components in leaves, up to $11.3 \%$ in inflorescences), heptacosane (up to $7.1 \%$ in leaves, up to $7.6 \%$ in inflorescences), stigmasterol (up to $8.1 \%$ in leaves, up to $2.2 \%$ in inflorescences), $\gamma$-sitosterol (up to $6.9 \%$ in leaves, up to $2.2 \%$ in inflorescences), $\beta$-amyrine (up to $4.1 \%$ in leaves, up to $5.8 \%$ in inflorescences), $\beta$-caryophyllene (up to $2.6 \%$ in leaves, up to $1.5 \%$ in inflorescences), caryophyllene oxide (up to $4.4 \%$ in leaves, up to $2.9 \%$ in inflorescences), germacrene D (up to $13.8 \%$ in leaves, up to $9.7 \%$ in inflorescences) and $\alpha$-pinene (up to $3.3 \%$ in leaves, up to $2.4 \%$ in inflorescences).

The chemical composition of lipophilic extract of rhizomes significantly differs from that of leaves and inflorescences. The following compounds can be counted among the dominant ones occurring in rhizome extracts: diterpene of labdane type - 16-acetoxycarterochaetol (up to 45\%) and sesquiterpenes - 7- $\beta$-H-silphiperfol-5-ene (up to $9.1 \%$ ), 7 - $\alpha$-H-silphiperfol-5-ene (up to $10.7 \%$ ), $\delta$-elemene (up to $7.6 \%$ ), isocomene (up to $5.6 \%$ ), germacrene D (up to $5.2 \%$ ) and modhephene (up to $5.1 \%$ ).
\end{abstract}

KEY WORDS: Silphium L., Silphium perfoliatum L., Asteraceae, GC/MS, lipophilic fraction, sesquiterpenes.

\section{INTRODUCTION}

The paper presented here is a fragment of a multidisciplinary study cycle carried out by the author on a NorthAmerican species of Silphium L. genus (Asteraceae) (Weryszko-Chmielewska et al. 1999a, b; Wolski et al. 1999, 2000; Kowalski 2001, 2002, 2003; Kowalski, Wolski 2001, 2003a, b, in press - 2004 online; Kowalski, Wierciński 2003; Kowalski et al. 2005 - in press).

The aim of the present paper was to analyze the lipophilic fraction of leaves, inflorescences and rhizomes of the cup plant (Silphium perfoliatum L.) cultivated in the Lublin region as a potential herbal or fodder raw material. The research carried out is of an experimental nature and enables the future categorisation of Silphium varieties by their chemotaxonomy.

\section{MATERIALS AND METHODS}

\section{Plant material}

The study was conducted making use of leaves, inflorescences and rhizomes of Silphium perfoliatum L., originating from ten-year old plants (1998), grown in the Department of Botany, University of Agriculture and from twoyear old plants (1999-2000), grown in the Department of Vegetable and Medicinal Plants, University of Agriculture in Lublin. The leaves and inflorescences were collected in July, and rhizomes in October. They were dried in shade and air and then ground.

\section{Extraction}

Extracts for study were obtained by extraction of 40 g powdered raw material using petroleum ether $(1: 10)$ in Soxhlet's apparatus $\left(12 \mathrm{hrs}, 60^{\circ} \mathrm{C}\right)$. Extracts were filtered 
and subsequently subjected to GC/MS analysis. In addition, the contents of the dry ether extract were evaluated by mass after prior solvent evaporation at a temperature of $60^{\circ} \mathrm{C}$.

\section{$G C / M S$}

The GC/MS instrument ITS-40 (GC/ITMS - Finnigan MAT, USA) was used, with $30 \mathrm{~m} \times 0.25 \mathrm{~mm}$ i.d., DB-5 column (J\&W, USA), film thickness $0.25 \mathrm{~mm}$, carrier gas $\mathrm{He}$, injector temperature $-280^{\circ} \mathrm{C}$. A temperature gradient was applied $\left(35^{\circ} \mathrm{C}\right.$ for 2 minutes, then increased by $4^{\circ} \mathrm{C} / \mathrm{min}$ to $280^{\circ} \mathrm{C}$ ). The qualitative analysis was carried out on the basis of MS spectra which were compared with the spectra of the NIST library (1992) and the LIBR (TR) terpenes library provided by Finnigan MAT (1990), and data available in literature (Bohlmann, Jakupovic 1979, 1980; Bohlmann et al. 1977, 1979a, b; Careri et al. 2001; Killops, Frewin 1994; Kowalski, Wolski in press - 2004 online; Joulain, König 1998; Zalkow et al. 1977, 1978; SCRI 2003). Identity of the compounds was confirmed by their retention indices taken from literature and own data (Kowalski 2001; Menut et al. 1997; Weyerstahl et al. 1997; Joulain, König 1998; Kowalski, Wolski in press - 2004 online; Palá-Paúl et al. 2002; Tellez et al. 2002).

The retention index defines the analyte peak location on the chromatogram with regard to the n-alkanes:

$$
I_{X}=\left(t_{R x}-t_{R n}\right) \div\left(t_{R n+1}-t_{R n}\right) \times 100+100 n
$$

where: $t_{R x}$ - the analyte retention time, $t_{R n}-$ the retention time of the $n$-alkane with $n$-carbon atoms, $t_{R n+1}-$ the retention time of alkane with $\mathrm{n}+1$ carbon atoms and $\mathrm{t}_{\mathrm{Rn}} \leq \mathrm{t}_{\mathrm{Rx}}$ $\leq \mathrm{t}_{\mathrm{Rn}+1}$.

The quantitative composition of extracts was determined assuming the total of the particular extracts to constitute $100 \%$.

\section{RESULTS AND DISCUSSION}

Petroleum ether extracts from leaves, inflorescences and rhizomes differed organoleptically one from another. Extracts produced from rhizomes were the most viscous and were characterised by a transparent amber colour and an odour recalling coniferous tree resin. Extracts prepared from leaves and inflorescences had a fatty-resin consistence and undetermined odour, as well as a green colour in the case of leaves and yellowish-brown colour in the case of inflorescences. Moreover, the percentage of the extracted dry matter recalculated onto studied organic dry matter was at the following levels: $1.70 \%$ for leaves, $4.53 \%$ for inflorescences and $2.52 \%$ for rhizomes (Table 1 ).

GC/MS chromatograms of the components of the extracts from leaves, inflorescences and rhizomes of cup

TABLE 1. Percentage of the Silphium petroleum ether extract dry matter recalculated onto studied organic dry matter.

\begin{tabular}{lcccc}
\hline \multirow{2}{*}{ Organs } & \multicolumn{4}{c}{ Content of Silphium petroleum ether extract (\%DM) } \\
\cline { 2 - 5 } & 1998 & 1999 & 2000 & Mean \\
\hline Leaves & 1.62 & 1.55 & 1.93 & 1.70 \\
Inflorescences & 4.65 & 4.27 & 4.67 & 4.53 \\
Rhizomes & 2.62 & 2.37 & 2.52 & 2.52 \\
\hline
\end{tabular}
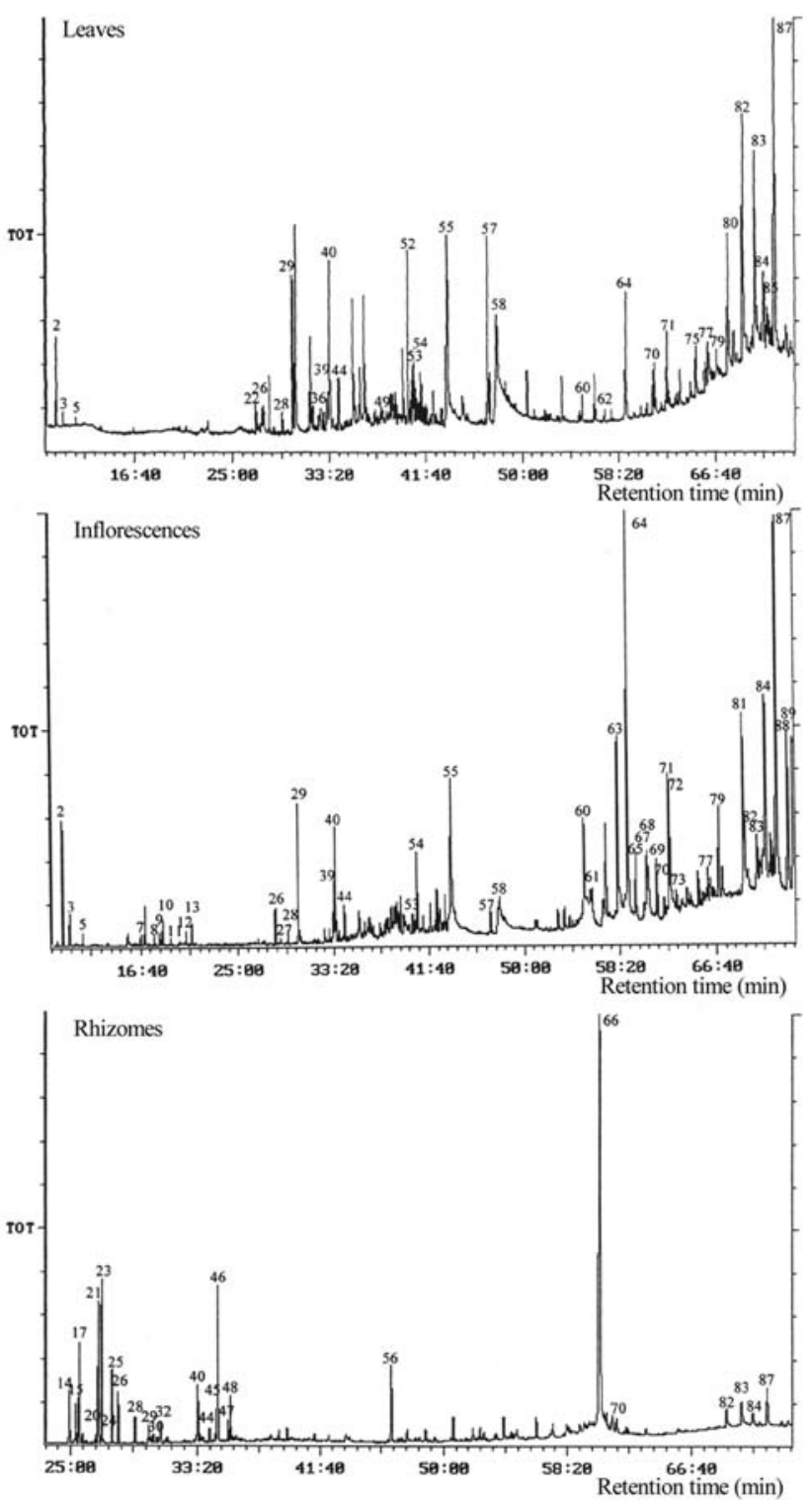

Fig. 1. GC/MS chromatograms of ingredients of lipophilic extracts made from leaves, inflorescences and rhizomes of cup-plant Silphium perfoliatum L. (1999) - individual compounds were marked in accordance to Table 2 .

plants are presented in Figure 1. Table 2 lists the percentage of particular components of studied materials.

GC/MS analysis of the extracts isolated from leaves and inflorescences showed that they were of similar composition. The following compounds were identified in both leaf and inflorescence extracts: $\alpha$-amyrine (triterpene alcohol), heptacosane (alkane), stigmasterol and $\gamma$-sitosterol (phytosterols), $\beta$-amyrine (triterpene alcohol). The occurrence of $\alpha$-amyrine - characteristic of plants producing resins and alkanes - in components of the cuticle covering the skin of the aboveground parts of plants, is noteworthy. Moreover, several chemical compounds that were earlier found in essential oils isolated from these raw materials (Wolski et al. 2000; Kowalski, Wolski in press - 2004 online), among others: $\beta$-caryophyllene caryophyllene oxide, germacrene $\mathrm{D}$ and $\alpha$-pinene, were present in the studied extracts.

The chemical composition of the petroleum ether extracts from rhizomes significantly differs from that of leaves and inflorescences. Labdane type diterpene (16-aceto- 
TABLE 2. The percentage of main components of lipophilic extracts from leaves, inflorescences and rhizomes of the cup-plant (Silphium perfoliatum L.) using petroleum ether.

\begin{tabular}{|c|c|c|c|c|c|c|c|c|c|c|c|c|}
\hline \multirow{3}{*}{ No. } & \multirow{3}{*}{ Compound } & \multirow{3}{*}{$\begin{array}{c}\text { Reten- } \\
\text { tion } \\
\text { index }\end{array}$} & \multicolumn{9}{|c|}{ Percentage share of the ingredients of lipophilic extracts (\%) } & \multirow{3}{*}{$\begin{array}{l}\text { Refe- } \\
\text { rence }\end{array}$} \\
\hline & & & \multicolumn{3}{|c|}{ Leaves } & \multicolumn{3}{|c|}{ Inflorescences } & \multicolumn{3}{|c|}{ Rhizomes } & \\
\hline & & & 1998 & 1999 & 2000 & 1998 & 1999 & 2000 & 1998 & 1999 & 2000 & \\
\hline 1 & tricyclene & 919.2 & - & - & 0.1 & - & tr. & 0.1 & - & - & - & $\mathrm{N}$ \\
\hline 2 & $\alpha$-pinene & 931.2 & 0.2 & 1.5 & 3.3 & 0.3 & 1.2 & 2.4 & - & - & - & $\mathrm{N}$ \\
\hline 3 & camphene & 945.8 & 0.2 & 0.2 & 0.5 & - & 0.3 & 0.4 & - & - & - & $\mathrm{N}$ \\
\hline 4 & sabinene & 971.5 & - & - & tr. & - & - & tr. & - & - & - & $\mathrm{N}$ \\
\hline 5 & $\beta$-pinene & 974.7 & 0.2 & 0.1 & - & 0.2 & 0.1 & 0.2 & - & - & - & $\mathrm{N}$ \\
\hline 6 & limonene & 1029.1 & tr. & - & tr. & tr. & tr. & tr. & - & - & - & $\mathrm{N}$ \\
\hline 7 & $\alpha$-pinene oxide & 1100.4 & 0.2 & - & 0.2 & 0.2 & 0.1 & 0.2 & - & - & - & $\mathrm{N}$ \\
\hline 8 & $\alpha$-campholenal & 1130.4 & - & - & 0.1 & - & 0.1 & - & - & - & - & $\mathrm{N}$ \\
\hline 9 & trans-pinocarveol & 1142.8 & - & - & 0.1 & 0.1 & 0.1 & - & - & - & - & $\mathrm{N}$ \\
\hline 10 & trans-verbenol & 1149.7 & - & - & 0.4 & 0.2 & 0.3 & 0.3 & - & - & - & $\mathrm{N}$ \\
\hline 11 & pinocarvone & 1168.1 & - & - & 0.1 & 0.1 & 0.1 & tr. & - & - & - & $\mathrm{N}$ \\
\hline 12 & myrtenal + myrtenol & 1202.1 & - & - & 0.2 & 0.1 & 0.2 & 0.1 & - & - & - & $\mathrm{N}$ \\
\hline 13 & verbenone & 1216.7 & 0.2 & - & 0.4 & 0.2 & 0.3 & 0.3 & - & - & - & $\mathrm{N}$ \\
\hline 14 & 7- $\alpha$-H-silphiperfol-5-ene & 1327.8 & 0.2 & - & - & 0.2 & - & - & 10.7 & 1.9 & 5.4 & 2,7 \\
\hline 15 & $\delta$-elemene & 1340.6 & tr. & - & - & 0.1 & - & - & 0.2 & 0.1 & 7.6 & 7 \\
\hline 16 & 7- $\beta$-H-silphiperfol-5-ene & 1343.6 & 0.2 & - & - & tr. & - & - & 5.7 & - & 9.1 & 2,7 \\
\hline 17 & n.i. & 1347.3 & - & - & - & - & - & - & 2.6 & 4.0 & - & \\
\hline 18 & silphiperfola-5,7 (14)-diene & 1361.3 & - & - & - & - & - & - & 0.9 & 0.1 & - & 7 \\
\hline 19 & $\alpha$-copaene & 1379.6 & 0.3 & - & 0.2 & 0.3 & tr. & 0.2 & 0.3 & - & - & 7 \\
\hline 20 & silphiperfol-6-ene & 1379.8 & $\operatorname{tr}$. & - & - & - & - & - & 0.7 & 0.3 & 0.7 & $2,7,11$ \\
\hline 21 & modhephene & 1384.7 & - & - & - & - & - & - & 2.1 & 5.1 & 1.2 & 3,17 \\
\hline 22 & $\beta$-bourbonene & 1389.2 & 1.2 & 0.5 & tr. & $\operatorname{tr}$. & - & - & - & - & - & 7 \\
\hline 23 & isocomene & 1391.9 & - & - & - & - & - & - & 2.4 & 5.6 & 0.7 & 4,11 \\
\hline 24 & $\beta$-elemene & 1395.8 & 0.3 & - & - & 0.2 & - & - & - & 0.1 & 0.1 & 16 \\
\hline 25 & $\beta$-isocomene & 1412.3 & - & - & - & - & - & - & 0.5 & 2.6 & 0.3 & 7 \\
\hline 26 & $\beta$-caryophyllene & 1424.8 & 2.6 & 1.2 & 1.2 & 1.5 & 0.5 & 1.0 & 0.6 & 1.8 & 2.0 & $7,15,11$ \\
\hline 27 & trans- $\alpha$-bergamotene & 1439.2 & 0.4 & tr. & 0.1 & 0.1 & 0.1 & 0.1 & - & - & - & 7 \\
\hline 28 & $\alpha$-humulene & 1459.8 & 0.7 & 0.5 & 0.3 & 0.4 & 0.2 & 0.2 & 1.2 & 0.9 & 2.4 & 11,15 \\
\hline 29 & germacrene D & 1487.5 & 13.5 & 3.2 & 4.3 & 9.1 & 1.7 & 3.6 & 0.5 & 0.6 & 5.2 & 7,15 \\
\hline 30 & $\delta$-selinene & 1497.4 & - & - & - & - & - & - & 0.7 & 0.4 & - & 7 \\
\hline 31 & germacrene B & 1499.2 & 0.2 & - & tr. & 0.1 & - & - & - & - & - & 7 \\
\hline 32 & $\beta$-bisabolene & 1512.9 & - & - & - & - & - & - & 1.4 & 0.8 & 0.7 & 7 \\
\hline 33 & $\gamma$-cadinene & 1516.5 & tr. & - & $\operatorname{tr}$. & - & - & - & tr. & tr. & - & 7 \\
\hline 34 & $\delta$-cadinene & 1529.9 & 0.3 & - & $\operatorname{tr}$ & 0.2 & tr. & - & 0.3 & tr. & - & 7 \\
\hline 35 & $\gamma$-elemene & 1566.2 & - & - & - & - & - & - & tr. & tr. & - & 7 \\
\hline 36 & (E)-nerolidol & 1568.1 & 0.8 & 0.1 & tr. & 0.1 & tr. & tr. & - & - & - & $\mathrm{N}$ \\
\hline 37 & n.i. & 1576.4 & 0.4 & 0.4 & 0.2 & 0.2 & 0.2 & 0.2 & - & tr. & - & 10 \\
\hline 38 & 4 - $\beta$-hydroksygermacra-1 (10),5-diene & 1584.6 & - & - & tr. & - & tr. & tr. & - & tr. & - & 10 \\
\hline 39 & spathulenol & 1587.6 & 1.5 & 1.1 & 0.5 & 0.7 & 0.4 & 0.5 & tr. & - & - & 11 \\
\hline 40 & caryophyllene oxide & 1593.1 & 1.8 & 4.4 & 3.1 & 1.2 & 2.9 & 2.8 & 3.0 & 1.8 & 0.3 & $\mathrm{~N}$ \\
\hline 41 & n.i. & 1596.4 & 1.0 & 0.4 & 0.4 & 0.5 & 0.3 & 0.4 & 0.2 & - & - & 10 \\
\hline 42 & salvial-4 (14)-en-1-one & 1604.1 & 0.1 & 0.1 & 0.2 & 0.1 & 0.1 & - & 0.2 & 0.4 & - & 10 \\
\hline 43 & globulol & 1610.7 & - & - & - & - & - & 0.2 & - & - & - & 12 \\
\hline 44 & humulene epoxide II + $\beta$-oplopenone & 1620.3 & 0.9 & 1.8 & 1.0 & 0.6 & 0.8 & 0.9 & 2.9 & 0.6 & - & 7 \\
\hline 45 & 2,3,5,9-tetramethyl-tricyclo-[6.3.0.0E1,5]-undek-2-en-4-one & 1635.1 & - & - & - & - & - & - & 5.0 & 0.2 & 0.2 & $\mathrm{~N}$ \\
\hline 46 & n.i. ${ }^{a}$ & 1640.0 & 0.3 & tr. & 0.2 & 0.5 & - & 0.1 & 4.0 & 5.9 & 2.6 & 10 \\
\hline 47 & aplotaxene & 1664.1 & - & - & - & - & - & - & 0.4 & 0.7 & 0.6 & 9 \\
\hline 48 & n.i. & 1669.6 & - & - & - & - & - & - & 0.5 & 1.7 & 1.0 & 10 \\
\hline
\end{tabular}


TABLE 2. Cont.

\begin{tabular}{|c|c|c|c|c|c|c|c|c|c|c|c|c|}
\hline \multirow{3}{*}{ No. } & \multirow{3}{*}{ Compound } & \multirow{3}{*}{$\begin{array}{l}\text { Reten- } \\
\text { tion } \\
\text { index }\end{array}$} & \multicolumn{9}{|c|}{ Percentage share of the ingredients of lipophilic extracts (\%) } & \multirow{3}{*}{$\begin{array}{l}\text { Refe- } \\
\text { rence }\end{array}$} \\
\hline & & & \multicolumn{3}{|c|}{ Leaves } & \multicolumn{3}{|c|}{ Inflorescences } & \multicolumn{3}{|c|}{ Rhizomes } & \\
\hline & & & 1998 & 1999 & 2000 & 1998 & 1999 & 2000 & 1998 & 1999 & 2000 & \\
\hline 49 & oplopanone & 1752.1 & 0.3 & 0.5 & - & 0.2 & 0.1 & - & - & - & - & 9 \\
\hline 50 & oplopenone & 1754.0 & $\operatorname{tr}$. & - & tr. & - & - & - & - & - & - & 9 \\
\hline 51 & n.i. & 1780.8 & 0.4 & 0.6 & 0.5 & 0.3 & 0.4 & 0.7 & - & - & - & \\
\hline 52 & n.i. & 1838.5 & 1.1 & 3.5 & 0.2 & 0.7 & 0.3 & 0.2 & - & - & - & \\
\hline 53 & 6,10,14-trimethyl-2-pentadecanone & 1847.1 & 0.9 & 0.6 & 0.3 & 0.2 & 0.2 & 0.4 & - & - & - & $\mathrm{N}$ \\
\hline 54 & n.i. & 1853.5 & 1.7 & 1.3 & 0.8 & 1.1 & 1.1 & 0.9 & - & - & - & 10 \\
\hline 55 & hexadecanoic acid & 1970.1 & 9.2 & 2.0 & 4.0 & 7.6 & 3.7 & 4.2 & - & - & - & $\mathrm{N}$ \\
\hline 56 & n.i. & 2093.6 & - & - & - & - & - & - & 5.7 & 3.5 & - & \\
\hline 57 & phytol & 2115.9 & 2.9 & 5.1 & 0.5 & 0.5 & 0.3 & 0.6 & - & - & - & 14 \\
\hline 58 & (Z,Z,Z)-9,12,15-octadecatrienoic acid methyl ester & 2155.8 & 11.2 & 5.8 & 0.6 & 4.3 & 0.8 & 1.8 & - & - & - & $\mathrm{N}$ \\
\hline 59 & n.i. & 2188.0 & - & - & - & - & - & - & - & - & 1.6 & $\mathrm{~N}$ \\
\hline 60 & pentacosane & 2495.5 & 0.6 & 0.5 & 2.3 & 1.3 & 2.0 & 2.5 & - & - & - & $\mathrm{N}$ \\
\hline 61 & docosanoic acid methyl ester & 2530.9 & - & - & 1.5 & 0.5 & 0.8 & 1.5 & - & - & - & $\mathrm{N}$ \\
\hline 62 & hexacosane & 2595.3 & 0.7 & 0.2 & - & - & - & - & - & - & - & $\mathrm{N}$ \\
\hline 63 & n.i. & 2653.0 & tr. & - & 5.7 & 5.0 & 3.4 & 5.9 & - & - & - & $\mathrm{N}$ \\
\hline 64 & heptacosane & 2695.6 & 1.9 & 6.3 & 7.1 & 7.3 & 6.9 & 7.6 & - & - & - & $\mathrm{N}$ \\
\hline 65 & tetracosanic acid methyl ester & 2737.0 & 0.6 & - & 2.6 & 2.2 & 0.9 & 2.6 & - & - & - & $\mathrm{N}$ \\
\hline 66 & 16-acetoxycarterochaetol & 2786.6 & - & - & - & - & - & - & 32.1 & 31.7 & 45.0 & 1 \\
\hline 67 & n.i. & 2789.0 & - & - & 1.1 & 1.0 & 1.4 & 1.0 & - & - & - & \\
\hline 68 & octacosane & 2794.5 & 0.3 & tr. & 0.7 & 0.7 & 0.8 & 0.8 & - & - & - & $\mathrm{N}$ \\
\hline 69 & n.i. & 2795.0 & - & - & 0.9 & 1.0 & 1.3 & 0.9 & - & - & - & \\
\hline 70 & squalene & 2838.0 & 1.0 & 1.2 & 0.9 & 0.9 & 0.8 & 0.8 & 0.3 & 0.4 & - & 13 \\
\hline 71 & nonacosane & 2900.0 & 1.1 & 1.5 & 2.6 & 4.2 & 2.1 & 2.6 & - & - & - & $\mathrm{N}$ \\
\hline 72 & n.i. & 2909.0 & 0.4 & - & 2.1 & 0.4 & 1.5 & 2.0 & - & - & - & \\
\hline 73 & hexacosanoic acid methyl ester & 2940.0 & 0.2 & tr. & 0.7 & 0.8 & 0.3 & 0.7 & - & - & - & $\mathrm{N}$ \\
\hline 74 & triacontane & 3000.0 & 0.2 & $\operatorname{tr}$ & - & 0.9 & $\operatorname{tr}$ & - & 0.5 & - & - & $\mathrm{N}$ \\
\hline 75 & n.i. & 3052.0 & 0.6 & 1.5 & 0.4 & $\operatorname{tr}$ & 0.6 & - & - & - & - & \\
\hline 76 & $\beta$-tocopherol & 3076.1 & - & - & - & 0.1 & 0.1 & - & - & - & - & $\mathrm{N}$ \\
\hline 77 & hentriacontane & 3100.0 & 0.3 & 0.6 & 0.8 & 0.4 & 0.5 & 0.7 & - & - & - & $\mathrm{N}$ \\
\hline 78 & octadecanoic acid methyl ester & n.d. & - & - & 0.3 & - & - & 0.3 & - & - & - & $\mathrm{N}$ \\
\hline 79 & vitamin $\mathrm{E}$ & n.d. & 0.3 & 0.4 & 1.5 & 0.9 & 1.4 & 1.4 & - & - & - & $\mathrm{N}$ \\
\hline 80 & n.i. & n.d. & 1.0 & 5.3 & - & - & - & - & - & - & - & \\
\hline 81 & n.i. & n.d. & - & - & - & 0.4 & 3.3 & 4.2 & - & - & - & \\
\hline 82 & stigmasterol & n.d. & 7.5 & 8.1 & 2.4 & 1.6 & 1.9 & 2.2 & 1.0 & 1.0 & 0.6 & $\mathrm{~N}, 5$ \\
\hline 83 & $\gamma$-sitosterol & n.d. & 5.1 & 6.9 & 1.7 & 1.1 & 1.2 & 0.9 & 1.3 & 1.5 & 0.4 & $\mathrm{~N}, 5$ \\
\hline 84 & $\beta$-amyrine & n.d. & 2.0 & 2.9 & 4.1 & 3.9 & 5.8 & 3.8 & 0.5 & 0.9 & 0.4 & $\mathrm{~N}, 6,8$ \\
\hline 85 & n.i. & n.d. & - & 1.0 & - & - & 0.9 & - & - & 0.1 & - & \\
\hline 86 & n.i. & n.d. & - & 0.3 & - & - & 1.2 & - & - & - & - & \\
\hline 87 & $\alpha$-amyrine & n.d. & 11.8 & 13.9 & 6.9 & 10.1 & 11.3 & 6.3 & 2.0 & 2.5 & 2.6 & $\mathrm{~N}, 6,8$ \\
\hline 88 & n.i. & n.d. & 0.3 & 0.4 & 1.9 & 1.9 & 3.8 & 1.7 & 0.3 & 0.3 & - & \\
\hline 89 & n.i. & n.d. & tr. & tr. & - & tr. & 3.9 & - & tr. & 0.3 & - & \\
\hline 90 & n.i. & n.d. & - & 1.3 & - & - & 1.8 & - & - & - & - & \\
\hline 91 & n.i. & n.d. & - & tr. & - & - & 1.2 & - & - & - & - & \\
\hline 92 & n.i. & n.d. & - & 0.6 & - & - & 4.5 & - & - & 1.0 & - & \\
\hline
\end{tabular}

„n.i.” - not identified; „tr.” - trace $(<0.1 \%)$; ,n.d.” - not determined; a - spectrum similar to that of spathulenol; N - NIST library and terpene library LIBR (Finnigan MAT); 1) Bohlmann, Jakupovic 1979; 2) Bohlmann, Jakupovic 1980; 3) Bohlmann et al. 1979a; 4) Bohlmann et al. 1977; 5) Careri et al. 2001; 6) Chosson et al. 2003; 7) Joulain, König 1998; 8) Killops, Frewin 1994; 9) Kowalski 2001; 10) Kowalski, Wolski in press - 2004 online; 11) Menut et al. 1997; 12) Palá-Paúl et al. 2002; 13) SCRI 2003; 14) Tellez et al. 2002; 15) Weyerstahl et al. 1997; 16) Zalkow et al. 1977; 17) Zalkow et al. 1978. 
xycarterochaetol) and sesquiterpene compounds (7- $\beta-\mathrm{H}-$ -silphiperfol-5-ene, $\delta$-elemene, isocomene, silphiperfol-6-ene, germacrene D, modhephene) dominated in rhizome extracts. Moreover, the presence of compounds occurring in oils isolated from the raw material was found. Previously, Bohlmann and Jakupovic $(1979,1980)$ had found that extracts made from Silphium perfoliatum L. rhizomes contained sesquiterpene hydrocarbons, including: aplotaxene, germacrene, eudesmane and guayane derivatives, modhephene, silphinene, silphiperfol-6-ene, 7- $\alpha$-H-silphiperfol-5-ene, 7- $\beta$-H-silphiperfol-5-ene, isocomene. Labdane type diterpenes and carterochaetol derivatives were a remarkable group of isoprenoid compounds in rhizomes. Our results are similar to the above data.

It is noteworthy that the percentage of particular components of the extracts obtained from the above and belowground parts of the cup plant varied depending on the plant's age and the study year, which can be accounted for by fluctuations in physiological and environmental factors. As regards the quality, the extracts studied showed no differences which were dependent on the year of experiment. Comparing the volatility of compounds isolated from leaves, inflorescences and rhizomes of the cup plant, it is demonstrated that volatile (low-molecular) compounds were present only in the aboveground organs (e.g. tricyclene, $\alpha$-pinene, camphene, $\beta$-pinene, $\mathrm{p}$-cymene, limonene, $\alpha$-campholenal, cis-verbenol, trans-pinocarveol, trans-verbenol, pinocarvone, myrtenal, myrtenol and verbenone).

The presented paper describes for the first time the composition of total lipophilic fraction (petroleum ether) of leaves, inflorescences and rhizomes of Silphium perfoliatum L. obtained from two- and ten-year old plants in cultivation experiments carried out in the period 1998-2000. The results may be useful for the evaluation of plants in relation to their biological activity, which will be discussed in the next paper.

\section{LITERATURE CITED}

BOHLMANN F., JAKUPOVIC J. 1979. Neue Labdan-Derivate und Sesquiterpene aus Silphium-Arten. Phytochemistry 18: 1987-1992.

BOHLMANN F., JAKUPOVIC J. 1980. Neue Sesquiterpen-Kohlenwasserstoffe mit anomalen Kohlenstoffgerüst aus SilphiumArten. Phytochemistry 19: 259-265.

BOHLMANN F., LE VAN N., PHAM T.V.C., JAKUPOVIC J., SCHUSTER A., ZABEL V., WATSON W.H. 1979a. $\beta$-Isocomen, ein neues Sesquiterpen aus Berkheya-Arten. Phytochemistry 18: 1831-1834.

BOHLMANN F., LE VAN N., PICKARDT J. 1977. Über ein anomales Sesquiterpenes aus Berkheya radula (Harv.) De Willd. Chem. Ber. 3777-3781.

BOHLMANN F., ZDERO C.H., KING R.M., ROBINSON H. 1979b. Neue Labdan-Derivate aus Carterothamnus anomalochaeta. Phytochemistry 18: 621-624.

CARERI M., ELVIRI L., MANGIA A. 2001. Liquid chromatography-UV determination and liquid chromatography-atmospheric pressure chemical ionization mass spectrometric characterization of sitosterol and stigmasterol in soybean oil. J. Chromatogr. A 935: 249-257.

CHOSSON E., VÉRITÉ P., BLANCKAERT A., SEGUIN E., LITAUDON M., SÉVENET T. 2003. Non polar compounds from the bark of Sarcomelicope follicularis. Biochemical Systematics and Ecology 31: 1185-1188.

FINNIGAN MAT, Terpene Library. USA (1990).
JOULAIN D., KÖNIG W.A. 1998. The atlas of spectral data of sesquiterpene hydrocarbons. E. B. - Verlag Hamburg.

KILLOPS S.D., FREWIN N.L. 1994. Triterpenoid diagenesis and cuticular preservation. Org. Geochem. 21: 1193-1209.

KOWALSKI R. 2001. Chemical analysis of above-ground and underground organs of Silphium perfoliatum L. Doctoral disertation. Univ. of Agric. Lublin, 1-219 (in Polish).

KOWALSKI R. 2002. Evaluation of oleanosides content in above and underground organs of Silphium perfoliatum L. Acta Scientiarum Polonorum Hortorum Cultus 1 (2): 5-15 (in Polish).

KOWALSKI R. 2003. Selected secondary metabolites in leaves, inflorescences, and rhizomes of Silphium perfoliatum L. - alternative herbal plant. Folia Hort. 15, 2, 203-209.

KOWALSKI R., WIERCIŃSKI J. 2003. Phenolic acids in leaves of three Silphium L. species. Pol. J. Food Sci. Nutr. 53, 17-20.

KOWALSKI R., WIERCIŃSKI J., MARDAROWICZ M. 2005. Essential oil in leaves and inflorescences of Silphium integrifolium Michx. J. Essent. Oil Res. (in press)

KOWALSKI R., WOLSKI T. 2001. Characteristics of growth and Development of Silphium perfoliatum L. in the first years of cultivation. Ann. Univ. Mariae Curie-Skłodowska sec. EEE 9, Supl., 311-317 (in Polish).

KOWALSKI R., WOLSKI T. 2003a. TLC and HPLC analysis of the phenolic acids in Silphium perfoliatum L. leaves, inflorescences and rhizomes. J. Planar Chromatogr. 16: 230-236.

KOWALSKI R., WOLSKI T. 2003b. Evaluation of phenolic acid content in Silphium perfoliatum L. leaves, inflorescences and rhizomes. EJPAU, http://www.ejpau.media.pl/series/volume6/issue1/horticulture/art-03.html.

KOWALSKI R., WOLSKI T. 2004 (online). The chemical composition of essential oils of Silphium perfoliatum L. Flav. Fragr. J. DOI 10.1002/ffj.1418 (in press)

MENUT C., LAMATY G., WEYERSTAHL P., MARSCHALL H., SEELMANN I., AMVAM ZOLLO P.H. 1997. Aromatic plants of tropical Central Africa. Part XXXI.\# Tricyclic sesquiterpenes from the root essential oil of Echinops giganteus var. lelyi C. D. Adams. Flav. Fragr. J. 12: 415-421.

NIST/EPA/NIH. 1992. Mass Spectral Library. USA.

PALÁ-PAÚL J., PÉREZ-ALONSO M.J., VELASCO-NEGUERUELA A., SANZ J. 2002. Analysis by gas chromatographymass spectrometry of the volatile components of Ageratina adenophora Spreng., growing in the Canary Islands. J. Chromatogr. A 947: 327-331.

SCRI, Mass spectra of some miscellaneous lipophilic components. Scotland (2003): http://www.lipid.co.uk/inflores/ms/ ms21/file.pdf

TELLEZ M.R., KHAN I.A., KOBAISY M., SCHRADER K.K., DAYAN F.E., OSBRINK W. 2002. Composition of the essential oil of Lepidium meyenii (Walp.). Phytochemistry 61: 149-155.

WERYSZKO-CHMIELEWSKA E., KOWALSKI R., WOLSKI T. 1999a. Silphium perfoliatum L. A new alternative plant. Part I. Morphological and anatomical investigation. Zesz. Probl. Post. Nauk Rol. 468: 497-505 (in Polish).

WERYSZKO-CHMIELEWSKA E., MICHOŃSKA M., WOLSKI T., KOWALSKI R. 1999b. Comparison of morphological features of flowers in three Silphium species with consideration of pollen presenters and pollen grains. Bibl. Frag. Agr. 6: 103-112 (in Polish).

WEYERSTAHL P., MARSCHALL H., SCHRÖDER M., WAHLBURG H.CH., KAUL V.K. 1997. The sesquiterpene fraction of the essential oil of Artemisia laciniata Willd. Flav. Fragr. J. 12: 315-325.

WOLSKI T., KOWALSKI R., MARDAROWICZ M. 2000. Chromatographic analysis of essential oil occurring in inflorescences, leaves and rhizomes of Silphium perfoliatum L. Herba Pol. 46 (4): 235-242.

WOLSKI T., KOWALSKI R., MARDAROWICZ M., WERYSZKO-CHMIELEWSKA E. 1999. Silphium perfoliatum L. 
A new alternative plant. Part II. Phytochemical analysis. Zesz. Probl. Post. Nauk Rol. 468, 507-517 (in Polish).

ZALKOW L.H., HARRIS III R.N., VAN DENVER D., BER-

TRAND J.A. 1977. Isocomene: a novel sesquiterpene from Isocoma Wrightii. X-Ray Crystal Structure of the corresponding Diol. J. C. S. Chem. Comm. 456-457.
ZALKOW L.H., HARRIS III R.N., VAN DENVER D. 1978. Modhephene: a sesquiterpenoid carbocyclic [3.3.3]propellane. X-Ray Crystal Structure of the corresponding Diol. J. C. S. Chem. Comm. 420-421. 